\title{
Action-Dependent Photobiomodulation on Health, Suboptimal Health, and Disease
}

\author{
Timon Cheng-Yi Liu, ${ }^{1}$ Long Liu, ${ }^{2}$ Jing-Gang Chen, ${ }^{1}$ Peng Zeng, ${ }^{1}$ and Xiang-Bo Yang ${ }^{1,3}$ \\ ${ }^{1}$ Laboratory of Laser Sports Medicine, South China Normal University, University Town, Guangzhou 510006, China \\ ${ }^{2}$ Department of Chemistry and Biology, College of Science, National University of Defense Technology, Changsha 410073, China \\ ${ }^{3}$ MOE Key Laboratory of Laser Life Science and Institute of Laser Life Science, College of Biophotonics, \\ South China Normal University, Guangzhou 510631, China
}

Correspondence should be addressed to Timon Cheng-Yi Liu; liutcy@scnu.edu.cn

Received 22 February 2014; Accepted 29 March 2014; Published 24 April 2014

Academic Editor: Quan-Guang Zhang

Copyright (C) 2014 Timon Cheng-Yi Liu et al. This is an open access article distributed under the Creative Commons Attribution License, which permits unrestricted use, distribution, and reproduction in any medium, provided the original work is properly cited.

\begin{abstract}
The global photobiomodulation (PBM) on an organism was studied in terms of function-specific homeostasis (FSH) and scalefree functional network in this paper. A function can be classified into a normal function in its FSH and a dysfunctional function far from its FSH. An FSH-specific stress (FSS) disrupting an FSH can also be classified into a successful stress in its FSS-specific homeostasis (FSSH) and a chronic stress far from its FSSH. The internal functions of an organism can be divided into essential, special nonessential, and general nonessential functions. Health may be defined as a state of an organism in which all the essential and special nonessential functions are normal or their stresses are successful. Suboptimal health may be defined as a state of a disease-free organism in which only some special nonessential functions are dysfunctional in comparison with its healthy state. Disease may be defined as a state of an organism which is not in both health and suboptimal health. The global PBM of health, suboptimal health, or disease suggested that the PBM may depend on the organism action.
\end{abstract}

\section{Introduction}

Unsustainable cost increases threaten the global health care system, and further progress is stymied more by societal than technological factors [1]. Conventional medical practice has been "reactive" (doctor takes part when disease appears). However, Sobradillo et al. [2] found that the theoretical (scale-free networks and complex systems), technological (high efficiency "omic" technologies), and conceptual (biology systems) advances throughout the last decade allowed us to anticipate in the transition to an "anticipatory" medicine known as "P4 medicine" (standing for personalized, predictive, preventive and participatory medicine). In fact, both the functional medicine [3] and traditional Chinese medicine (TCM) $[4,5]$ are also "anticipatory" medicines mainly from the functional viewpoint [6]. Constitutionbased TCM strategies [7] and photobiomodulation (PBM) [8] in disease prevention and treatment are consistent with the current proposed P4 medical mode. At its foundation,
P4 medicine is about quantifying health and demystifying disease [1]. However, the current definition of health is vague and incomplete [1]. The exact definitions of health, suboptimal health, and disease and their PBM mechanism are examined in this paper from the viewpoints of functions and scale-free networks.

\section{Functional Negative Feedback}

Negative feedback is common in biological processes and acts to optimize the activity of a circuit in the presence of alleles with altered activities [9], which can maintain a system's stability to internal and external perturbations. Function-specific homeostasis (FSH) is a negative-feedback response of a biosystem to maintain the function-specific conditions inside the biosystem so that the function is perfectly performed $[8,10-12]$. The quality of an FSH may be called a functional fitness of the maintained normal function. 
The fitness has been used to discuss function performance by Nowak et al. [13], but the functional fitness was not defined. A function in and far from its FSH is called a "normal/dysfunctional function," respectively. The transition of a dysfunctional function to a normal function is called "to be normalized." A normal function is better performed than all the dysfunctional functions so that the normal function is locally the best performed function. These phenomena can be explained by using the Arndt-Schulz law [14] when, after an initial peak response, the marginal efficacy declines as dosage increases. FSH can finely extend this curve into a plateau.

A functional fitness is maintained by its normal function. Endurance exercise is any activity that uses large muscle groups can be performed continuously and is rhythmic and aerobic in nature. To maintain a cardiovascular fitness, this exercise should be performed at a frequency of 3 to 5 days per week, at an intensity of $60 \%$ to $90 \%$ of maximum heart rate (HRmax) or $50 \%$ to $85 \%$ of HRmax reserve, and at a duration of 20 to $60 \mathrm{~min}$ [15]. An inverse relationship exists between physical fitness and resting heart rate, body weight, percent body fat, serum cholesterol, triglycerides, glucose, and systolic blood pressure. In addition, exercise training increases the high-density lipoprotein portion of total cholesterol.

A normal function can resist internal/external disturbance under its threshold. It is resilient or robust. Higher functional fitness offers higher threshold. Using skill level as an example, Hancock [16] examined the effect of individual skill level on task performance in transient extreme heat and found that individuals who were skillful at the task were better able to withstand the detrimental effect of the stress exposure than their unskilled counterparts.

An FSH can be disrupted by an FSH-specific stress (FSS). The FSS is also a function of a biosystem [11, 12]. A normal/dysfunctional FSS is called a "successful/chronic stress," respectively and has been referred to as being "antifragile/fragile" by Taleb [17]. Taleb [17] hypothesized that the antifragileness should be beyond the resilience or robustness of a normal function. The resilience resists shocks and stays the same, but the antifragileness gets better and better. Moreover, hormetic (in small doses) stress $[17,18]$ is another kind of successful stress. A successful stress upgrades a normal function, but a chronic stress destroys a normal function. These phenomena are explained by the ArndtSchulz Law [14], the Yerkes-Dodson law in psychology [19], or nonlinear relationships commonly described as being Uor J-shaped or inverted U- or J-shaped [18].

The simplest, most obvious, and best appreciated mechanism for buffering genetic variation is redundancy [9]. Redundancy can play an important role in hormesis [20] and antifragileness [17]. It also enhances performance. As an example, elite athletes often undertake altitude training to improve sea-level athletic performance. Robertson et al. [21] found that a combined approach of live high/train low plus train high elicited greater enhancements in physiological capacities when compared with the train high approach. Taleb [17] illustrated the redundancy-mediated antifragileness with a barbell-style approach. His preferred strategy was to be both hyperconservative and hyperaggressive at the same time. Instead of doing steady and moderate exercise daily, he suggested that it should be better to do a low-effort exercise such as walking slowly most of the time, while occasionally expending extreme effort. Redundancy enhances the cellular function $[11,12]$. The $m$ genes performing the same function, respectively, are called redundant genes with one another. Each redundant gene may activate a normal function-specific signal transduction (NSP) [12]. The $m$ NSPs are called redundant pathways with one another. As it has been hypothesized [12], the full activation of each NSP may maintain the firstorder normal function in its NSP-specific microenvironment (NSM), and the synergistic full activation of one NSP and its $\mathrm{n}-1$ redundant pathways may maintain the $n$ th-order normal function $(n=2,3, \ldots, m)$ in its NSM.

Let $Q$ be the quality of a FSH or the functional fitness of a normal function. An organism has many kinds of functions which include internal functions and external activities. Their normal ones are being maintained by their respective FSH $\left\{\mathrm{FSH}_{i}, i=1,2, \ldots, n\right\}$ and their respective quality $\left\{\mathbf{Q}_{i}, i=1,2, \ldots, n\right\}$. Their performance is accompanied by various rhythms; higher functional fitness gives higher rhythm fitness. Janiak and kedziora [22] investigated the effect of exercise on the diurnal changes of electromechanical systolic time (QS2) in healthy men with different physical fitness and found that the effect of exercise on the circadian rhythm of QS2 was dependent on the level of physical fitness. After exercise, the circadian rhythm QS2 was presented in the human with higher physical fitness, whereas it was not evident in human with lower physical fitness. On the other hand, the enhancement of normal rhythms can upgrade the related normal functions or activities. Zhao et al. [23] found that, when a PBM improved sleep and melatonin, it also enhanced endurance performance.

Let $Q \max =\max \left\{Q_{i}, i=1,2, \ldots, l\right.$. Let $F \max$ and $F \operatorname{maxSH}$ denote the corresponding function/activity and its homeostasis, respectively. The $Q \max$ increases when a person grows but decreases when the person ages. The age at which the $Q \max$ is the highest, $Q p$, is called the prime age $h$. As growing and aging are symmetrical with each other, the most possible lifespan $y$ of a person may be $2 h+1[8,24]$. Obviously, the higher the $Q p$ and/or the slower the increase in $Q$ max, the longer the expected lifespan.

\section{Scale-Free Networks}

Many complex systems can be viewed as networks, in which nodes represent system elements and edges correspond to interactions between those elements. Many results suggest that networks from different domains but in the same category may be more similar to one another than previously thought [25]. Among the popular networks, the scale-free networks are extremely heterogeneous, their topology being dominated by a few highly connected nodes (essential nodes) which link the rest of the less connected nodes (nonessential nodes) to the system [2]. A network diameter is defined as the shortest pathway averaged over all pairs of nodes. To investigate whether networks displayed a similar error tolerance, Jeong et al. [26] performed computer simulations 
on the metabolic network of E. coli. Upon removal of the essential nodes the diameter increased rapidly, illustrating the special role of these nodes in maintaining a constant metabolic network diameter. However, when randomly chosen nonessential nodes were removed-mimicking the consequence of random mutations of catalysing enzymesthe average distance between the remaining nodes was not affected, indicating a striking insensitivity to random errors. Indeed, in silico and in vivo mutagenesis studies indicated remarkable fault tolerance upon removal of a substantial number of metabolic enzymes from the E. coli metabolic network [27].

All scale-free networks are sparse [28]. It suggested that their essential nodes should be sparse. A cell far from its FSH has many partially activated signal transduction pathways, but a cell in its FSH has sparse fully activated signal transduction pathways [29] so that there are NSPs [12]. There are many factors that contribute to maintaining a FSH state, but FSH-essential factors are very sparse [8]. Examples are a wide range of reprogramming of biological systems using overexpression of only one to five transcription factors [30] and a white-collar worker performing normal office work with very little brain material [31]. As shown in the next section, an organism has five essential internal functions and only one essential external activity.

\section{Function Network}

The scale-free networks dominate all aspects of human health and disease [2]. Generally, the nodes are genes, proteins, cells, or organisms. As presented in this paper the nodes are functions in the internal body or activities in the external body. Either the internal functions or external activities of an organism can be essential or nonessential according to the scale-free network properties. It depends on its importance in lifespan. The essential ones play important roles in healthy lifespan. The nonessential ones do not significantly affect the healthy lifespan under their respective threshold. They are further classified into the special nonessential ones which significantly affect the lifespan of nonhealthy body and the general nonessential ones which do not significantly affect the lifespan of nonhealthy body under their respective threshold.

4.1. Internal Functions. Western mainstream medicine including P4 medicine studies an organism mainly from anatomical viewpoint, but functional medicine [3] and TCM [6] study an organism mainly from the functional viewpoint. The internal function network has often been discussed from the TCM $[4,5]$ viewpoint.

An organism has many internal functions [3-5]. Among them, the five yin zang functions, heart function, liver function, spleen function, lung function, and kidney function, should be essential functions according to TCM. Navarrete et al. [32] analyzed organ mass data for 100 mammalian species, including 23 primates. They found that, controlling for fat-free body mass, there was significant positive mass correlation between heart and lungs or liver, between lungs and kidneys, liver, or spleen, between kidneys and liver or spleen, or between liver and spleen. In TCM, the heart rate belongs to the essential heart function. Jensen et al. [33] have done a prospective cohort study, the Copenhagen Male Study, a longitudinal study of healthy middle-aged employed men, and found that elevated resting heart rate was a risk factor for mortality independent of physical fitness, leisure-time physical activity, and other major cardiovascular risk factors. Xiong et al. [34] investigated physiological parameters during practicing simplified 24-form T'ai chi ch'uan (TCC) in 10 young high-level male TCC athletes and 10 ordinary-level male TCC practitioners with similar age and body size. They found significantly higher energy expenditure, heart rate, oxygen uptake, and tidal volume in the high-level group than the ordinary-level group during TCC performance. In TCM, gamma glutamyltransferase (GGT) and alkaline phosphatase (ALP) belong to the essential liver function. Kunutsor et al. [35] conducted a systematic review and meta-analysis of published prospective cohort studies evaluating the associations of baseline levels of these enzymes with all-cause mortality in general populations. Nineteen unique cohort studies with aggregate data on over 9.24 million participants and 242, 953 all-cause mortality outcomes were included. They found that the pooled relative risks ( $95 \%$ confidence intervals) for all-cause mortality per $5 \mathrm{U} / \mathrm{L}$ increment in GGT and ALP levels were 1.07 (1.04-1.10) and 1.03 (1.01-1.06), respectively. In TCM, forced expiratory volume in 1 second (FEV1) and peripheral blood leukocyte count belong to the essential lung and spleen functions. Weiss et al. [36] explored the relation of leukocyte count and FEV1 to total mortality in the Normative Aging Study population in the Boston, Massachusetts, area. The sample for the current analysis consisted of 1,956 men who underwent the baseline Normative Aging Study examination during 1961-1969. Subjects ranged in age from 21 to 80 years of age at the time of entry. A total of 170 deaths occurred over the 30 years of follow-up. They found that age, FEV1, and peripheral blood leukocyte count were the three most important predictors of increased mortality in this cohort, independent of cigarette smoking. In TCM, plasma fibrinogen belongs to the essential kidney function. Stack et al. [37] identified 9, 184 subjects at the age of 40 years and over from the Third National Health and Nutrition Examination Survey (1988-1994) with vital status assessed through 2006. They found that the adjusted hazard ratio (HR) per $1 \mu \mathrm{mol} / \mathrm{L}(34 \mathrm{mg} / \mathrm{dL})$ increase in fibrinogen was 1.07 (95\% confidence interval (CI) 1.04-1.09) for total mortality and 1.06 (95\% CI 1.03-1.09) for cardiovascular mortality in multivariate analysis.

Qi function, blood function, body fluid functions, meridian functions, acupoint functions, and the six yang fu functions, gallbladder function, stomach function, small intestine function, large intestine function, bladder function, and triple energizer function, should be special nonessential functions according to TCM. In TCM, haemoglobin belongs to the nonessential blood function. Skjelbakken et al. [38] examined 6541 men aged between 20 and 49 years in 1974 in a prospective, population-based study from the municipality of Troms $\varnothing$, Northern Norway, for 20 years of follow-up and found a U-shaped relationship between quintiles of haemoglobin and total mortality. In the above-mentioned 
cohort of Weiss et al. [36], other covariates examined included forced vital capacity, height, body mass index, systolic and diastolic blood pressure, and total cholesterol, but age, FEV1, and peripheral blood leukocyte count were the three most important predictors of increased mortality.

The extraordinary $f u$ functions such as brain function, marrow function, bone functions, blood vessel functions, gallbladder function, uterus function, pericardium function, life gate function, and essence chamber function should be general nonessential functions according to TCM. In TCM, brain mass belongs to the general nonessential brain function. Navarrete et al. [32] analyzed brain size and organ mass data for 100 mammalian species, including 23 primates. They found that, controlling for fat-free body mass, brain size was not negatively correlated with the mass of the digestive tract or any other expensive organ. In TCM, reproduction belongs to the general nonessential essence chamber function. Castration, which removes the source of male sex hormones, prolongs male lifespan in many animals [39]. Min et al. [40] studied the genealogy records of Korean eunuchs and determined the lifespan of 81 eunuchs and found that the average lifespan of eunuchs was $70.0 \pm 1.76$ years, which was 14.4-19.1 years longer than the lifespan of noncastrated men of similar socioeconomic status.

In TCM, the neurological functions except consciousness belong to the general nonessential brain function. Feuillet et al. [31] reported the case of an anatomically "brainless" white-collar worker who functioned much better than could be expected with so little brain material. The 44-year-old worker presented a 2-week history of mild left leg weakness. At the age of 6 months, he had undergone a ventriculoatrial shunt, because of postnatal hydrocephalus of unknown cause. When he was 14 years old, he developed ataxia and paresis of the left leg, which resolved entirely after shunt revision. His neurological development and medical history were otherwise normal. He was a married father of two children and worked as a civil servant. On neuropsychological testing, he proved to have an intelligence quotient (IQ) of 75: his verbal IQ was 84 and his performance IQ was 70 . Computer tomography (CT) showed severe dilatation of the lateral ventricles. Magnetic resonance imaging revealed massive enlargement of the lateral, third, and fourth ventricles, a very thin cortical mantle and a posterior fossa cyst. Feuillet et al. [31] diagnosed a noncommunicating hydrocephalus, with probable stenosis of Magendie's foramen. The leg weakness improved partly after neuroendoscopic ventriculocisternostomy but soon recurred; however, after a ventriculoperitoneal shunt was inserted, the findings on neurological examination became normal within a few weeks. The findings on neuropsychological testing and CT did not change.

4.2. External Activities. A person may have many external activities [41, 42]. An average adult's personal $F \max$ as discussed in Section 2 should be his/her occupational work [43]. Li [43] has collected 104 dead scientists or celebrities and found that the Pearson correlation coefficients of the prime occupation age with the lifespan and the prime age $h$ discussed in Section 2 were 0.901 and 0.906 , respectively, and there was a positive linear relationship between the prime occupation age and the lifespan. Moreover, the higher the $Q p$ or/and the slower the increases in $Q$ max, the longer the expected lifespan. Therefore, the occupational work should be the only essential activity. While studying engagement outcomes worldwide, Gallup [44] discovered a correlation between employees' engagement levels at work and their physical health. Low engagement may lead to low skill level [45]. In another study by Tjepkema et al. [46], a 15\% sample of 1991 Canadian Census respondents aged 25 years or older was linked to 16 years of mortality data (1991-2006). The study analyzed 2.3 million people aged 25 to 64 years at cohort inception, among whom there were 164, 332 deaths during the follow-up period, and found that mortality gradients by occupational skill level were evident for most causes of death.

The observations have been directly supported by butterfly experiments conducted by Niitepõld and Hanski [47]. They analyzed the repeatability of measurements of peak flight metabolic rate (MR (peak)) throughout the life of the Glanville fritillary butterfly (Melitaea cinxia). They found that measurements of MR (peak) showed significant repeatability and there was a significant positive correlation between MR (peak) and lifespan in all three experiments: in the laboratory, under field conditions, and in a laboratory experiment with repeated flight treatments.

These findings are also supported by a study on competitive sports by Teramoto and Bungum [48]. They reviewed elite endurance (aerobic) athletes and mixed-sports (aerobic and anaerobic) athletes and found that they survive longer than the general population, as indicated by lower mortality and higher longevity. For example, a former career elite athlete would be protected from both type 2 diabetes and impaired glucose tolerance in later life [49] and an athlete doing high- but not low-intensity cycling may resist diesel exhaust exposure [50].

Wiseman [51] suggested that physical actions may be the quickest, easiest, and most powerful way to instantly change how one thinks and feels. This suggested that subjective wellbeing (SWB) should be a nonessential activity. There is a longstanding idea that happiness causes people to live longer, healthier lives. However, convincing evidence that SWB contributes to longevity and health has not been available [52]. Chida and Steptoe [53] have reviewed systematically prospective, observational cohort studies of the association between positive well-being and mortality using metaanalytic methods and suggested that positive psychological well-being has a favorable effect on survival in both healthy and diseased populations. Many other kinds of studies also concluded that happiness has a positive causal effect on longevity and physiological health [52]. However, other studies did not support it. Wiest et al. [54] have examined differential effects of life satisfaction (LS), positive affect (PA), and negative affect (NA) on mortality in a conjoint analysis using data of German adults between the ages of 40 and 85 years $(N=3,124)$. They found that LS and PA predicted mortality over and above sociodemographic factors and physical health. However, this effect diminished when it included self-rated health and physical activity. NA was not associated with mortality. Although PA predicted mortality in older adults $(65+)$ even after controlling for self-rated health 
and physical activity, no SWB indicator predicted mortality when controlling for covariates in middle-aged participants.

The nonessential activities can be further classified into the special ones and the general ones. Kahneman et al. [41] have assessed the range of mean enjoyment ratings for the lowest and highest levels of selected person and job characteristics after controlling for other job features or for other personal features for 909 employed women in USA. The ranges of both constant pressure to work quickly (definitely yes or definitely not) work episodes and sleep quality (very bad or very good) home episodes ranked the first. The ones of requires specialized education/training (definitely yes or definitely not) work episodes, importance of religion (not at all or very important) home episodes, and household income (less than $\$ 30,000$ or more than $\$ 90,000$ ) home episodes ranked the second. The ones of constant pressure to work quickly (definitely yes or definitely not) home episodes, at risk of being laid off (definitely yes or definitely not) work episodes, and marital status (divorced or married) home episodes ranked the third. These findings paralleled the observation that life circumstances have surprisingly little influence on global reports of life satisfaction [41]. These results suggested that sleep, one of the first rank activities, should be a special nonessential activity, but the other activities of second or third rank should be general nonessential ones. The nonessential sleep was supported by the U-shaped relationship between survival and actigraphically measured sleep durations [55] and between the hazard ratios for all-cause mortality and sleep duration [56]. It has been found that melatonin administration during daytime does not have any acute (1-2 h) effects either on the maximal jumping ability of adult men or on the maximal strength [57] and daily locomotor activity levels were similar between wild-type and circadian Clock mutant mice throughout the training protocol [58]. Moreover, sleep may be a special nonessential activity. Garde et al. [59] found that short sleep duration is a risk factor for ischemic heart disease mortality among middle-aged and elderly men, particularly those using tranquilizers/hypnotics on a regular or even a rare basis, but not among men not using tranquilizers/hypnotics.

SWB can be represented by health-related quality of life (HRQOL) [60]. HRQOL can be also represented by the EuroQol questionnaire (EQ-5D) [61]. Healthy Japan 21 (Japanese National Health Promotion in the 21st Century) was started in 2000 to promote extension of healthy life expectancy and improve HRQOL. Fujikawa et al. [61] described HRQOL of Japanese subjects using the EQ-5D and investigated the influence of social background, health-related behaviors, and chronic conditions on HRQOL using representatives in Takamatsu, Japan. They found that EQ-5D scores decreased with age, particularly for respondents who were unemployed or retired. Adjusting to sex and age, the results showed that age, unemployment/retirement, feeling severe stress, and musculoskeletal and gastrointestinal diseases were significantly associated with decreased HRQOL. Conversely, sufficient sleep (7-8 h/day) and having a hobby were significantly associated with increased HRQOL. It should be pointed out that there was no significant association between exercise and HRQOL although exercise is one of hobby activities. These results supported the special nonessential nature of sleep and suggested that a hobby is a special nonessential activity.

In TCM, nutrition corresponds to the nutritive qi function which belongs to a special nonessential function, and it should be a special nonessential activity. Many studies have found that there was a U-shaped relationship between body mass index (BMI) and mortality, morbidity, hazard risk, medical costs, physical fitness, subjective well-being, job strain, health-related quality of life, or other health-related outcomes. Goyal et al. [62] evaluated all-cause, cancer, and cardiovascular mortality risks associated with quintiles (Q1Q5) of serum antioxidant (vitamins $\mathrm{C}$ and $\mathrm{E}, \beta$-carotene, and selenium) and vitamin A levels, in 16,008 adult participants of The Third National Health and Nutrition Examination Survey (NHANES III; 1988-1994) and found that both vitamins $\mathrm{A}$ and $\mathrm{E}$ had $\mathrm{U}$-shaped associations with all-cause mortality.

\section{Organism Photobiomodulation}

There are two kinds of PBM [11, 12], direct and indirect PBM ( $\mathrm{dPBM}$ and $\mathrm{PBM})$. A dPBM can promote the normalization of a dysfunctional function [11] by promoting the activation of one partially activated NSP [12]. An iPBM can upgrade a normal function [11] maintaining a fully activated NSP by promoting the activation of one or more redundant pathways of the NSP and then the synergistic action of all the fully activated redundant NSPs [12]. Their mechanisms on health, suboptimal health, and disease were discussed in this section.

5.1. Health Promotion. Health is a state of complete physical, mental, and social well-being and not merely the absence of disease or infirmity according to the World Health Organization. Health is the state of an organism with undisturbed functional dynamic homeostasis providing optimum performance of organism functions to the extent necessary for productive relations of the organism with the environment according to Kryzhanovsky [63]. When discussing health, Taleb [17] thought that antifragileness should play an important role. As it has been pointed out in Section 2, the socalled antifragileness was just successful stress. In terms of FSH, FSSH, and scale-free functional network, health may be defined as a state of an organism in which its essential and special non-essential functions are normal or their stresses are successful. Its general nonessential functions may be dysfunctional or their stresses may be chronic. In this context, health can be of different levels because its normal functions can be of different levels and the phase transition of health can be discussed in view of the phase transition of its normal functions as discussed in our previous paper [12]. The higher the functional fitness of one of the essential or special nonessential functions is, the higher the health level is. In TCM, consciousness belongs to the essential five yin zang functions. Through kinesiological testing, Hawkins [64] has estimated the various levels of consciousness.

A global PBM can enhance health level by upgrading a normal function or activity in an PBM way or normalizing a dysfunctional function or activity in a dPBM way. The work of an athlete is doing sports. His/her performance represents 
his/her health level. Zhao et al. [23] have globally irradiated elite Chinese female basketball players wearing swimsuits with red light at $658 \mathrm{~nm}$ and $30 \mathrm{~J} / \mathrm{cm}^{2}$ for $30 \mathrm{~min}$ every night during 14 routine days including 12 training days with the following specifications: 2 hours of morning training, 2 hours of afternoon training, and no training on Sunday. They found that the 14-day whole-body irradiation improved sleep, serum melatonin level, and endurance performance. LealJunior et al. [65] have performed a systematic review with meta-analysis to investigate the effects of the PBM applied before exercises. They found that 12 randomized controlled trials (RCTs) irradiated phototherapy before exercise among which 10 RCTs reported significant improvement in the main outcome measures related to performance. The time until exhaustion increased significantly compared to placebo by $4.12 \mathrm{~s}$ and the number of repetitions increased by $5.47 \mathrm{after}$ phototherapy. They concluded that the PBM improved muscular performance and accelerated recovery mainly when applied before exercise.

Based on the theory of constitution in TCM, the human health can be classified into nine constitutions including a balanced constitution and eight unbalanced constitutions, yang-deficiency, yin-deficiency, qi-deficiency, phlegmwetness, wetness-heat, stagnant blood, depression, and inherited special constitutions [66, 67]. For the balanced constitution, the functional fitness of normal essential and special nonessential functions matches with one another. For the unbalanced constitution, the functional fitness of some normal functions is lower than the balanced one so that the functional fitness of normal essential and special nonessential functions does not match with one another. A global PBM can enhance health level by upgrading a normal function in an iPBM way so that it can transform unbalanced constitutions into the balanced constitution. We have analyzed the published epidemiological investigation of nine constitutions in nine provinces in China [68] according to province latitudes and found that sunlight may transform the unbalanced constitutionssuch as qi-deficiency, yangdeficiency, yin-deficiency, phlegm-wetness, or depression into the balanced constitution. Obviously, the skin-decayed sunlight can play an $\mathrm{iPBM}$ role so that it can enhance the functional fitness.

5.2. Suboptimal Health Rehabilitation. Although suboptimal health has been mentioned as early as in 1979 [69], its exact definition has been left unresolved. In terms of FSH, FSSH, and scale-free functional network, the suboptimal health may be defined as a state of disease-free organism in which some special nonessential functions are lightly dysfunctional or their stresses are lightly chronic in comparison with health definition.

Suboptimal health is often self-limited [11, 70]. Health can resist low-level disturbance under the threshold, but high-level disturbance of some special nonessential functions above their respective threshold may result in suboptimal health. Suboptimal health can be completely self-recovered if the high-level disturbance is eliminated. A dPBM can promote the normalization of dysfunctional special nonessential functions and possibly even at the high-level disturbance.
For example, intranasal low-intensity laser therapy (ILILT) has been used in health care applications for hyperlipidemia, blood hyperviscosity, and insomnia [8]. In a study, it treated high blood coagulation status in healthy pregnant women at term [8].

The SWB or HRQOL of an organism in suboptimal health is lower than the one in health. The higher the health level is, the higher the SWB or HRQOL is. Jia and Lubetkin [71] have examined a 1993-2006 monthly behavioral risk factor surveillance system (BRFSS) random sample and found that the worst physical health was during the winter and the best physical health was during the summer. Oswald and $\mathrm{Wu}$ [72] have examined a 2005-2008 BRFSS random sample of 1.3 million U.S. citizens and found that southern citizens were averagely happier than northern citizens. It might partially be due to the intensity of southern or summer sunlight being high enough for the skin-decayed sunlight to promote the rehabilitation of citizens in suboptimal health in a dPBM way or enhance the health level of citizens in health in an iPBM way. Of the sample of 7979 individuals, being representative of the Finnish general population aged 30 and over, Grimaldi et al. [73] found that the HRQoL was influenced by both the seasonal changes in mood and behavior and the illumination experienced indoors. Greater seasonal changes and poor illumination indoors were associated with more severe mental ill-being.

5.3. Disease Treatment. According to Kryzhanovsky [63], disease is a state of an organism with disturbed functional dynamic homeostasis and inability to perform to a necessary extent, with the functions providing productive relations with the environment. In this paper, the disease may be defined as a state far from its health or suboptimal health. It may be classified into two kinds, the essential and nonessential disease. The essential disease such as myocardial infarction is a state of an organism in which some of the essential functions are dysfunctional or their stresses are chronic. A nonessential disease such as diabetes is a state of an organism in which some special nonessential functions are heavily dysfunctional or their stresses are heavily chronic so that the functional fitness of some nonessential functions is too low for healthy survival.

The initial phase of a disease is often self-limited [11, 70]. Health can resist low-level disturbance, but high-level disturbance may result in disease. The initial phase of a disease can be completely self-recovered if the high-level disturbance is eliminated. A dPBM can promote the normalization of one dysfunctional function if the self-recovery process is delayed. For example, ILILT has positive clinical applications in mild cognitive impairment, Alzheimer's disease, Parkinson's disease, schizophrenia, pain relief, stroke, depression, inflammation, coronary heart disease, myocardial infarction, and cerebral palsy [8], and potential applications in hypertension, vascular dementia, cancer, diabetes, influenza, olfactory dysfunction, myopia, withdrawal symptoms, and renal failure [8], and its possible applications in upper respiratory tract infection, asthma, osteoarthritis, exercise-induced muscle damage, wound, traumatic brain injury, and osteoporosis [74]. 
The network of condition-specific functions of a patient with a disease which cannot be self-limited is also a scalefree network. The functions can be classified into the essential and nonessential functions. The dysfunctional nonessential function may be self-limited, but the dysfunctional essential function cannot be self-limited and its normalization may be promoted with a dPBM. For fibrosis [75], the nonessential oxidative stress or inflammation may be self-limited, but the delayed essential tissue self-regeneration can be promoted with a dPBM.

5.4. Action-Dependent Organism Photobiomodulation. Cellular functions and their PBM on cells are decided by their microenvironment [12]. For example, mitotic cells in their respective proliferation-specific homeostasis inactivate DNA double-strand break repair [76]. A PBM on the irradiated tissue should be decided by the tissue essential functions. For example, a dPBM on fibrosis can promote the delayed essential tissue self-regeneration [75]. A global PBM on an organism should be decided by the organism action, especially the normal action in its action-specific homeostasis (ASH). A PBM may modulate any functions. As actions may be the quickest, easiest, and most powerful way to instantly change how one thinks and feels [51], the organism action or its ASH would inhibit function PBM, a PBM which modulate a function, unless the function PBM is needed for action normalization, ASH maintenance, or its fitness enhancement. The higher the ASH fitness is, the stronger the resistance of the ASH is. For example, an athlete doing high- but not low-intensity cycling may resist diesel exhaust exposure [50]. Therefore, a global PBM on an organism may promote the normalization of a dysfunctional action in a dPBM way or upgrade the normal action in an $\mathrm{PBM}$ way and then modulate organism health, suboptimal health, and disease.

The PBM modulated actions may be circadian rhythms. Circadian rhythms prepare an organism for daily external activities or internal functions. The fitness enhancement of circadian rhythms can upgrade the related normal activities or functions. A PBM on circadian rhythm recovery or enhancement may promote the recovery or enhancement. The circadian rhythm protein period 2 (Per2) has been implicated in cardiac adaptation to limited oxygen availability. Eckle et al. [77] found that recovery of Per2 in the heart by exposing mice to bright light resulted in the transcriptional induction of glycolytic enzymes and Per2-dependent cardioprotection from ischemia. Melatonin entrains many aspects of the biological clock via activation of specific G-proteincoupled integral membrane melatonin receptors [78]. ILILT promoted melatonin recovery has found its applications in treating Alzheimer's disease, Parkinson's disease, poststroke depression and insomnia $[8,74]$ and its possible applications in treating wound, traumatic brain injury, and osteoporosis [74].

The PBM modulated actions may be sleep, preactivity warming, or postactivity cooling. Anafi et al. [79] found that sleep enhanced organ-specific molecular functions and that it had a ubiquitous role in reducing cellular metabolic stress in both brain and peripheral tissues. Their data suggested a novel role for sleep in synchronizing transcription in peripheral tissues. Night time is the due time for melatonin increase, which is triggered by the circadian rhythms [80]. Warmup exercise may increase physical fitness [81]. Therefore, the iPBM enhancement of the circadian rhythms fitness during night time [23] or warming time [65] can enhance exercise performance as has been discussed in Section 5.1.

The PBM modulated actions may be exercise. $\mathrm{Ng}$ et al. [82] found that successful aging in multivariate models was significantly associated with age $(\mathrm{OR}=0.90)$, female gender $(\mathrm{OR}=1.37)$, $\geq 6$ years of education $(\mathrm{OR}=2.31)$, better housing $(\mathrm{OR}=1.41)$, religious or spiritual beliefs $(\mathrm{OR}=$ $1.64)$, physical activities and exercise $(\mathrm{OR}=1.90)$, and low or no nutritional risk $(\mathrm{OR}=2.16)$. Liu et al. [83] found that treadmill exercise enhanced learning and memory function not only in wild-type mice but also in APP/PS1 transgenic mouse model of Alzheimer's disease paralleled by longterm potentiation. ILILT may be directly used to treat aging persons or patients with Alzheimer's disease [8] and may also be used to treat exercising elders or exercising patients with Alzheimer's disease, and the therapeutic effects of the latter approach may be better than the ones of the former approach.

The recoveries of suboptimal health or disease need cellular factors by circadian rhythms. They also need cells, especially mesenchymal stem cells (MSCs), which has been supported by widely used cell therapy. The MSCs can be transplanted. They can also be increased in situ with a PBM [84]. Sprague-Dawley rats underwent experimental myocardial infarction (MI). Tuby et al. [84] have compared the tibia irradiation with heart irradiation of low-intensity laser irradiation (Ga-Al-As diode laser, power density $10 \mathrm{~mW} / \mathrm{cm}^{2}$, for 100 seconds). They found that the tibia irradiation was more effective than heart irradiation. They further found that the tibia irradiation was mediated by bone marrow-derived MSCs.

\section{Discussions}

In the above-mentioned studies, the health, suboptimal health, and disease of an organism have been defined in terms of FSH, FSSH, and scale-free functional network, and their global PBM have been discussed, respectively. The global $\mathrm{PBM}$ on an organism may depend on the organism action, but the corresponding studies are very preliminary.

A patient or animal usually does nothing else while doing suboptimal health or disease treatment with a PBM. The therapeutic effects may be enhanced if the patient or animal does something to aid the PBM treatment. For example, the patient can sleep, listen to music, draw a picture, read materials, or exercise. The best alternative may be to be at his/her $F$ max as discussed in Section 2. The $F$ max is often achieved while performing the occupational work [43], the unique essential external activity as discussed in Section 4.2. As Tjepkema et al. [46] have found, mortality gradients by occupational skill level were evident for most causes of death. The higher the occupational skill level is, the lower the mortality for most causes of death is. In this context, the therapeutic effects of the global PBM on an organism may be 
mediated and enhanced by its modulation on the organism action. The laser device for in situ activity modulation may be ILILT, wrist low-intensity laser therapy, or interdigital lowintensity laser therapy.

Among other therapies offering global PBM, sunlight is the simplest. The skin-decayed sunlight may play an important role in outdoor effects on action performance according to the action-dependent mechanism of a global PBM. The hypothesis that there are added beneficial effects to be gained from performing physical activity outdoors in natural environments is very appealing and has generated considerable interest [85]. Compared with exercising indoors, exercising in natural environments has been associated with greater feelings of revitalization and positive engagement, decreases in tension, confusion, anger, and depression, and increased energy. Participants also reported greater enjoyment and satisfaction with outdoor activity and declared a greater intent to repeat the activity at a later date [85]. Athletes' times in $200 \mathrm{~m}$ indoor races are greater than in outdoor races [86]. The outdoor effects have been also observed in many animals or birds. Chen et al. [87] have investigated the effects of outdoor access on the growth performance and meat quality of broiler chickens and found that outdoor access had no effect on growth performance and yield traits but could improve the meat quality; birds reared with outdoor access from $36 \mathrm{~d}$ of age had better appearance and meat quality than those with outdoor access from $71 \mathrm{~d}$ of age.

The skin-decayed bright light may also play an important role in bright light intensification of affective responses [88] according to the action-dependent mechanism of a global PBM. Across six experiments, $\mathrm{Xu}$ et al. [88] found that light increased people's perception of ambient warmth, which in turn activates their hot emotional system, leading to intensified affective reactions-positive and negative-to different kinds of stimuli. Across different domains, ranging from feelings towards words to judgments of ad scripts and ad models for aggressiveness and sexiness, and to choice of food spiciness levels and consumptions of drinks, they found that light intensified both experienced and anticipated affective reactions, and consequently, influenced judgment and choice in a variety of contexts. Importantly, the awareness of a potential influence of ambient brightness on affective response did not result in a correction of its influence. The reality, based on their data, however, is that bright light intensifies positive or negative affective responses. For example, the participants consumed more of the favorable juice in bright rather than dimmed light, but less of the unfavorable juice; bright-light compared to dim light enhanced positive feelings toward the favorable drink and enhanced negative feelings toward the unfavorable drink; ambient brightness amplified affective reaction which impacts consumption; and people consumed more of favorable drinks and less of unfavorable drinks in bright light.

\section{Conclusions}

The health, suboptimal health, and disease of an organism may be defined in terms of FSH, FSSH, and scale-free functional network. Their global PBM may depend on the organism action so that the PBM modulated action may modulate PBM therapeutic effects.

\section{Conflict of Interests}

The authors declare that there is no conflict of interests regarding the publication of this paper.

\section{Authors' Contribution}

Timon Cheng-Yi Liu, Long Liu, and Jing-Gang Chen contributed equally to this work.

\section{Acknowledgments}

This work was supported by National Science Foundation of China (60878061, 10974061, and 11374107), Doctoral Fund of Ministry of Education of China (20124407110013), and Guangdong Scientific Project (2012B031600004).

\section{References}

[1] L. Hood and N. D. Price, "Demystifying disease, democratizing health care," Science Translational Medicine, vol. 6, no. 225, Article ID 225ed5, 2014.

[2] P. Sobradillo, F. Pozo, and Á. Agustí, "P4 medicine: the future around the corner," Archivos de Bronconeumologia, vol. 47, no. 1, pp. 35-40, 2011.

[3] D. S. Jones, Textbook of Functional Medicine, The Institute for Functional Medicine, Gig Harbor, Wash, USA, 2006.

[4] Z. Xie, On the Standard Nomenclature of Traditional Chinese Medicine, Foreign Languages Press, Beijing, China, 2003.

[5] P. C. Leung, C. C. Xue, and Y. C. Cheng, Eds., A Comprehensive Guide to Chinese Medicine, World Scientific, River Edge, NJ, USA, 2003.

[6] M. A. Hyman, "Systems of correspondence: functionality in traditional Chinese medicine and emerging systems biology," Alternative Therapies in Health and Medicine, vol. 12, no. 2, pp. 10-11, 2006.

[7] J. Wang, Q. Wang, L. Li et al., "Phlegm-dampness constitution: genomics, susceptibility, adjustment and treatment with traditional Chinese medicine," The American Journal of Chinese Medicine, vol. 41, no. 2, pp. 253-262, 2013.

[8] T. C.-Y. Liu and P. Zhu, Intranasal Low Intensity Laser Therapy, People’s Military Medical Press, Beijing, China, 2009.

[9] J. L. Hartman IV, B. Garvik, and L. Hartwell, "Cell biology: principles for the buffering of genetic variation," Science, vol. 291, no. 5506, pp. 1001-1004, 2001.

[10] T. C.-Y. Liu, R. Liu, L. Zhu, J. Q. Yuan, M. Hu, and S. H. Liu, "Homeostatic photobiomodulation," Frontiers of Optoelectronics in China, vol. 2, no. 1, pp. 1-8, 2009.

[11] T. C.-Y. Liu, Y. Y. Liu, E. X. Wei, and F. H. Li, "Photobiomodulation on stress," International Journal of Photoenergy, vol. 2012, Article ID 628649, 11 pages, 2012.

[12] T. C.-Y. Liu, D. Wu, L. Zhu, P. Zeng, L. Liu, and X. Yang, "Microenvironment dependent photobiomodulation on function-specific signal transduction pathways," International Journal of Photoenergy, vol. 2014, Article ID 904304, 8 pages, 2014. 
[13] M. A. Nowak, M. C. Boerlijst, J. Cooke, and J. M. Smith, "Evolution of genetic redundancy," Nature, vol. 388, no. 6638, pp. 167-170, 1997.

[14] F. Martius, "Das amdt-schulz grandgesetz," Munchener Medizinische Wochenschrift, vol. 70, pp. 1005-1006, 1923.

[15] L. T. Braun, "Exercise physiology and cardiovascular fitness," Nursing Clinics of North America, vol. 26, no. 1, pp. 135-147, 1991.

[16] P. A. Hancock, "The effect of skill on performance under an environmental stressor," Aviation, Space, and Environmental Medicine, vol. 57, no. 1, pp. 59-64, 1986.

[17] N. N. Taleb, Antifragile: Things That Gain from Disorder, Random House Press, New York, NY, USA, 2012.

[18] E. J. Calabrese, K. A. Bachmann, A. J. Bailer et al., "Biological stress response terminology: integrating the concepts of adaptive response and preconditioning stress within a hormetic dose-response framework," Toxicology and Applied Pharmacology, vol. 222, no. 1, pp. 122-128, 2007.

[19] R. M. Yerkes and J. D. Dodson, "The relation of strength of stimulus to rapidity of habit-formation," Journal of Comparative Neurology and Psychology, vol. 18, no. 5, pp. 459-482, 1908.

[20] A. I. Yashin, "Hormesis against aging and diseases: using properties of biological adaptation for health and survival improvement," Dose-Response, vol. 8, no. 1, pp. 41-47, 2010.

[21] E. Y. Robertson, P. U. Saunders, D. B. Pyne, C. J. Gore, and J. M. Anson, "Effectiveness of intermittent training in hypoxia combined with live high/train low," European Journal of Applied Physiology, vol. 110, no. 2, pp. 379-387, 2010.

[22] A. Janiak and J. Kedziora, "Exercise effect on the circadian rhythm of left ventricular systole in healthy men with higher and lower physical fitness," Acta Physiologica Polonica, vol. 33, no. 4, pp. 345-351, 1982.

[23] J. Zhao, Y. Tian, J. Nie, J. Xu, and D. Liu, "Red light and the sleep quality and endurance performance of Chinese female basketball players," Journal of Athletic Training, vol. 47, no. 6, pp. 673-678, 2012.

[24] T. C.-Y. Liu, L. Zhu, and X. B. Yang, "Longevity chance," Journal of Innovative Optical Health Sciences, vol. 7, no. 2, Article ID 1330006, 6 pages, 2013.

[25] J. P. Onnela, "Physics: flow of control in networks," Science, vol. 343, no. 6177, pp. 1325-1326, 2014.

[26] H. Jeong, B. Tombor, R. Albert, Z. N. Oltval, and A.-L. Barabásl, "The large-scale organization of metabolic networks," Nature, vol. 407, no. 6804, pp. 651-654, 2000.

[27] J. S. Edwards and B. O. Palsson, "The Escherichia coli MG1655 in silico metabolic genotype: its definition, characteristics, and capabilities," Proceedings of the National Academy of Sciences of the United States of America, vol. 97, no. 10, pp. 5528-5533, 2000.

[28] C. I. del Genio, T. Gross, and K. E. Bassler, "All scale-free networks are sparse," Physical Review Letters, vol. 107, no. 17, Article ID 178701, 4 pages, 2011.

[29] T. C.-Y. Liu, L. Zhu, and X. B. Yang, "Photobiomodulationmediated pathway diagnostics," Journal of Innovation in Optical Health Science, vol. 6, no. 1, Article ID 133000, 11 pages, 2013.

[30] F.-J. Müller and A. Schuppert, "Few inputs can reprogram biological networks," Nature, vol. 478, no. 7369, pp. E4-E5, 2011.

[31] L. Feuillet, H. Dufour, and J. Pelletier, "Brain of a white-collar worker," The Lancet, vol. 370, no. 9583, p. 262, 2007.

[32] A. Navarrete, C. P. van Schaik, and K. Isler, "Energetics and the evolution of human brain size," Nature, vol. 480, no. 7375, pp. 91-93, 2011.
[33] M. T. Jensen, P. Suadicani, H. O. Hein, and F. Gyntelberg, "Elevated resting heart rate, physical fitness and all-cause mortality: a 16-year follow-up in the Copenhagen Male Study," Heart, vol. 99, no. 12, pp. 882-887, 2013.

[34] K. Xiong, H. He, and G. X. Ni, "Effect of skill level on cardiorespiratory and metabolic responses during Tai Chi training," European Journal of Sport Science, vol. 13, no. 4, pp. 386-391, 2013.

[35] S. K. Kunutsor, T. A. Apekey, D. Seddoh, and J. Walley, "Liver enzymes and risk of all-cause mortality in general populations: a systematic review and meta-analysis," International Journal of Epidemiology, vol. 43, no. 1, pp. 187-201, 2014.

[36] S. T. Weiss, M. R. Segal, D. Sparrow, C. Wager, R. J. Crowell, and J. M. Samet, "Relation of FEV1 and peripheral blood leukocyte count to total mortality: the Normative Aging Study," American Journal of Epidemiology, vol. 142, no. 5, pp. 493-503, 1995.

[37] A. G. Stack, U. Donigiewicz, A. A. Abdalla et al., "Plasma fibrinogen associates independently with total and cardiovascular mortality among subjects with normal and reduced kidney function in the general population," Quarterly Journal of Medicine, 2014.

[38] T. Skjelbakken, T. Wilsgaard, O. H. Førde, E. Arnesen, and M.L. Løchen, "Haemoglobin predicts total mortality in a general young and middle-aged male population. The Tromsø Study," Scandinavian Journal of Clinical \& Laboratory Investigation, vol. 66, no. 7, pp. 567-576, 2006.

[39] H. M. Brown-Borg, "Hormonal regulation of longevity in mammals," Ageing Research Reviews, vol. 6, no. 1, pp. 28-45, 2007.

[40] K. J. Min, C. K. Lee, and H. N. Park, "The lifespan of Korean eunuchs," Current Biology, vol. 22, no. 18, pp. R792-R793, 2012.

[41] D. Kahneman, A. B. Krueger, D. A. Schkade, N. Schwarz, and A. A. Stone, "A survey method for characterizing daily life experience: the day reconstruction method," Science, vol. 306, no. 5702, pp. 1776-1780, 2004.

[42] M. A. Killingsworth and D. T. Gilbert, "A wandering mind is an unhappy mind," Science, vol. 330, no. 6006, p. 932, 2010.

[43] J. Li, The study of scientist lifespan based on the function-specific homeostasis theory [M.S. of science thesis], South China Normal University, 2012.

[44] State of the Global Workplace, http://www.gallup.com/strategicconsulting $/ 164735 /$ state-global-workplace.aspx?utm_source= WWW\&utm_medium=csm\&utm_campaign=syndicationutm.

[45] P. Belchior, M. Marsiske, S. Sisco, A. Yam, and W. Mann, "Older adults' engagement with a video game training program," Activities, Adaptation \& Aging, vol. 36, no. 4, pp. 269-279, 2012.

[46] M. Tjepkema, R. Wilkins, and A. Long, "Cause-specific mortality by occupational skill level in Canada: a 16-year follow-up study," Chronic Diseases and Injuries in Canada, vol. 33, no. 4, pp. 195-203, 2013.

[47] K. Niitepõld and I. Hanski, "A long life in the fast lane: positive association between peak metabolic rate and lifespan in a butterfly," The Journal of Experimental Biology, vol. 216, no. 8, pp. 1388-1397, 2013.

[48] M. Teramoto and T. J. Bungum, "Mortality and longevity of elite athletes," Journal of Science and Medicine in Sport, vol. 13, no. 4, pp. 410-416, 2010.

[49] M. K. Laine, J. G. Eriksson, U. M. Kujala et al., "A former career as a male elite athlete-does it protect against type 2 diabetes in later life?" Diabetologia, vol. 57, no. 2, pp. 270-274, 2014. 
[50] L. V. Giles, J. P. Brandenburg, C. Carlsten, and M. S. Koehle, "Physiological responses to diesel exhaust exposure are modified by cycling intensity," Medicine \& Science in Sports \& Exercise, 2014.

[51] R. Wiseman, Rip It up: The Radically New Approach to Changing Your Life, Conville \& Walsh Press, London, UK, 2012.

[52] B. S. Frey, "Happy people live longer," Science, vol. 331, no. 6017, pp. 542-543, 2011.

[53] Y. Chida and A. Steptoe, "Positive psychological well-being and mortality: a quantitative review of prospective observational studies," Psychosomatic Medicine, vol. 70, no. 7, pp. 741-756, 2008.

[54] M. Wiest, B. Schüz, N. Webster, and S. Wurm, "Subjective wellbeing and mortality revisited: differential effects of cognitive and emotional facets of well-being on mortality," Health Psychology, vol. 30, no. 6, pp. 728-735, 2011.

[55] D. F. Kripke, R. D. Langer, J. A. Elliott, M. R. Klauber, and K. M. Rex, "Mortality related to actigraphic long and short sleep," Sleep Medicine, vol. 12, no. 1, pp. 28-33, 2011.

[56] Y. Yeo, S. H. Ma, S. K. Park et al., "A prospective cohort study on the relationship of sleep duration with all-cause and diseasespecific mortality in the Korean Multi-Center Cancer Cohort Study," Journal of Preventive Medicine and Public Health, vol. 46, no. 5, pp. 271-281, 2013.

[57] A. A. Mero, M. Vähälummukka, J. J. Hulmi, P. Kallio, and A. von Wright, "Effects of resistance exercise session after oral ingestion of melatonin on physiological and performance responses of adult men," European Journal of Applied Physiology, vol. 96, no. 6, pp. 729-739, 2006.

[58] S. Pastore and D. A. Hood, "Endurance training ameliorates the metabolic and performance characteristics of circadian Clock mutant mice," Applied Physiology, vol. 114, no. 8, pp. 1076-1084, 2013.

[59] A. H. Garde, Å. M. Hansen, A. Holtermann, F. Gyntelberg, and P. Suadicani, "Sleep duration and ischemic heart disease and all-cause mortality: prospective cohort study on effects of tranquilizers/hypnotics and perceived stress," Scandinavian Journal of Work, Environment \& Health, vol. 39, no. 6, pp. 550558, 2013.

[60] R. A. Cummins, "Fluency disorders and life quality: subjective wellbeing vs. health-related quality of life," Journal of Fluency Disorders, vol. 35, no. 3, pp. 161-172, 2010.

[61] A. Fujikawa, T. Suzue, F. Jitsunari, and T. Hirao, "Evaluation of health-related quality of life using EQ-5D in Takamatsu, Japan," Environmental Health and Preventive Medicine, vol. 16, no. 1, pp. 25-35, 2011.

[62] A. Goyal, M. B. Terry, and A. B. Siegel, "Serum antioxidant nutrients, vitamin a, and mortality in US adults," Cancer Epidemiology, Biomarkers \& Prevention, vol. 22, no. 12, pp. 2202-2211, 2013.

[63] G. N. Kryzhanovsky, "Some categories of general pathology and biology: health, disease, homeostasis, sanogenesis, adaptation, immunity: new approaches and notions," Pathophysiology, vol. 11, no. 3, pp. 135-138, 2004.

[64] D. R. Hawkins, Power versus Force, Hay House, Carlsbad, Calif, USA, 2002.

[65] E. C. Leal-Junior, A. A. Vanin, E. F. Miranda, P. D. de Carvalho, S. dal Corso, and J. M. Bjordal, "Effect of phototherapy (lowlevel laser therapy and light-emitting diode therapy) on exercise performance and markers of exercise recovery: a systematic review with meta-analysis," Lasers in Medical Science, 2013.
[66] Q. Wang and S. Yao, "Molecular basis for cold-intolerant yangdeficient constitution of traditional chinese medicine," The American Journal of Chinese Medicine, vol. 36, no. 5, pp. 827834, 2008.

[67] J. Wang, Y. Li, C. Ni, H. Zhang, L. Li, and Q. Wang, "Cognition research and constitutional classification in chinese medicine," The American Journal of Chinese Medicine, vol. 39, no. 4, pp. 651660, 2011.

[68] Q. Wang and Y. Zhu, "Epidemiological investigation of constitutional types of Chinese medicine in general population: base on 21,948 epidemiological investigation data of nine provinces in China," China Journal of Traditional Chinese Medicine and Pharmacy, vol. 24, no. 1, pp. 7-12, 2009 (Chinese).

[69] R. G. Farmer and W. M. Michener, "Prognosis of Crohn's disease with onset in childhood or adolescence," Digestive Diseases and Sciences, vol. 24, no. 10, pp. 752-757, 1979.

[70] J. Bigelow, A Discourse on Self-Limited Diseases (1835), Kessinger, Whitefish, Mont, USA, 1999.

[71] H. Jia and E. I. Lubetkin, "Time trends and seasonal patterns of health-related quality of life among US adults," Public Health Reports, vol. 124, no. 5, pp. 692-701, 2009.

[72] A. J. Oswald and S. Wu, "Objective confirmation of subjective measures of human well-being: evidence from the USA," Science, vol. 327, no. 5965, pp. 576-579, 2010.

[73] S. Grimaldi, T. Partonen, S. I. Saarni, A. Aromaa, and J. Lönnqvist, "Indoors illumination and seasonal changes in mood and behavior are associated with the health-related quality of life," Health and Quality of Life Outcomes, vol. 6, article $56,2008$.

[74] T. C.-Y. Liu, D.-F. Wu, Z.-Q. Gu, and M. Wu, "Applications of intranasal low intensity laser therapy in sports medicine," Journal of Innovative Optical Health Sciences, vol. 3, no. 1, pp. $1-16,2010$.

[75] X. E. Li, L. Zhu, and T. C. Y. Liu, "Fibrosis inhibition of photobiomodulation promoted regeneration," Photomedicine and Laser Surgery, vol. 31, no. 10, pp. 505-506, 2013.

[76] A. Orthwein, A. Fradet-Turcotte, S. M. Noordermeer et al., "Mitosis inhibits DNA double-strand break repair to guard against telomere fusions," Science, 2014.

[77] T. Eckle, K. Hartmann, S. Bonney et al., "Adora2b-elicited Per2 stabilization promotes a HIF-dependent metabolic switch crucial for myocardial adaptation to ischemia," Nature Medicine, vol. 18, no. 5, pp. 774-782, 2012.

[78] A. F. Wiechmann and J. A. Summers, "Circadian rhythms in the eye: the physiological significance of melatonin receptors in ocular tissues," Progress in Retinal and Eye Research, vol. 27, no. 2, pp. 137-160, 2008.

[79] R. C. Anafi, R. Pellegrino, K. R. Shockley, M. Romer, S. Tufik, and A. I. Pack, "Sleep is not just for the brain: transcriptional responses to sleep in peripheral tissues," BMC Genomics, vol. 14, article 362, 2013.

[80] C. Cajochen, C. Jud, M. Münch, S. Kobialka, A. Wirz-Justice, and U. Albrecht, "Evening exposure to blue light stimulates the expression of the clock gene PER2 in humans," European Journal of Neuroscience, vol. 23, no. 4, pp. 1082-1086, 2006.

[81] S. A. Zeno, D. Purvis, C. Crawford, C. Lee, P. Lisman, and P. A. Deuster, "Warm-ups for military fitness testing: rapid evidence assessment of the literature," Medicine \& Science in Sports \& Exercise, vol. 45, no. 7, pp. 1369-1376, 2013.

[82] T. P. Ng, B. F. P. Broekman, M. Niti, X. Gwee, and E. H. Kua, "Determinants of successful aging using a multidimensional 
definition among chinese elderly in singapore," The American Journal of Geriatric Psychiatry, vol. 17, no. 5, pp. 407-416, 2009.

[83] H.-L. Liu, G. Zhao, K. Cai, H.-H. Zhao, and L.-D. Shi, "Treadmill exercise prevents decline in spatial learning and memory in APP/PS1 transgenic mice through improvement of hippocampal long-term potentiation," Behavioural Brain Research, vol. 218, no. 2, pp. 308-314, 2011.

[84] H. Tuby, L. Maltz, and U. Oron, "Induction of autologous mesenchymal stem cells in the bone marrow by low-level laser therapy has profound beneficial effects on the infarcted rat heart," Lasers in Surgery and Medicine, vol. 43, no. 5, pp. 401409, 2011.

[85] J. T. Coon, K. Boddy, K. Stein, R. Whear, J. Barton, and M. H. Depledge, "Does participating in physical activity in outdoor natural environments have a greater effect on physical and mental wellbeing than physical activity indoors? A systematic review," Environmental Science \& Technology, vol. 45, no. 5, pp. 1761-1772, 2011.

[86] A. Ferro and P. Floria, "Differences in 200-m sprint running performance between outdoor and indoor venues," The Journal of Strength and Conditioning Research, vol. 27, no. 1, pp. 83-88, 2013.

[87] X. Chen, W. Jiang, H. Z. Tan et al., "Effects of outdoor access on growth performance, carcass composition, and meat characteristics of broiler chickens," Poultry Science, vol. 92, no. 2, pp. 435-443, 2013.

[88] A. J. Xu and A. A. Labroo, "Incandescent affect: turning on the hot emotional system with bright light," Journal of Consumer Psychology, vol. 24, no. 2, pp. 207-216, 2014. 

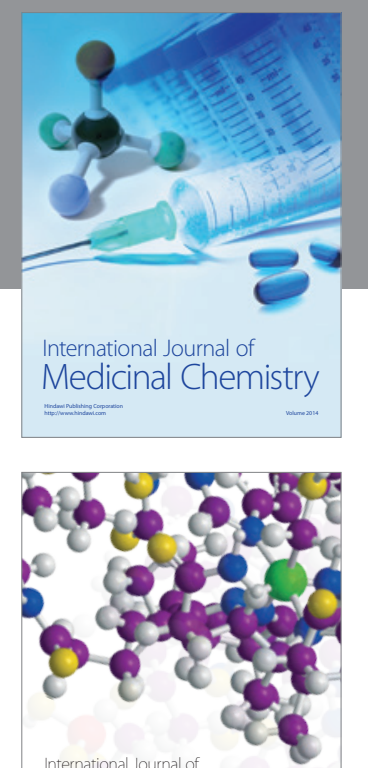

\section{Carbohydrate} Chemistry

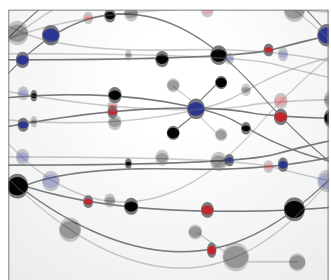

The Scientific World Journal
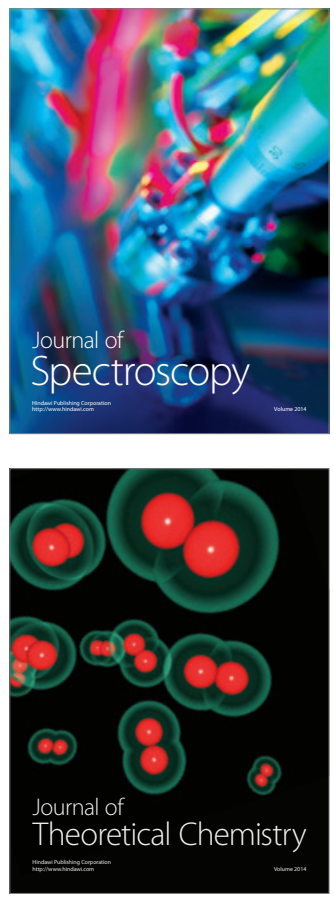
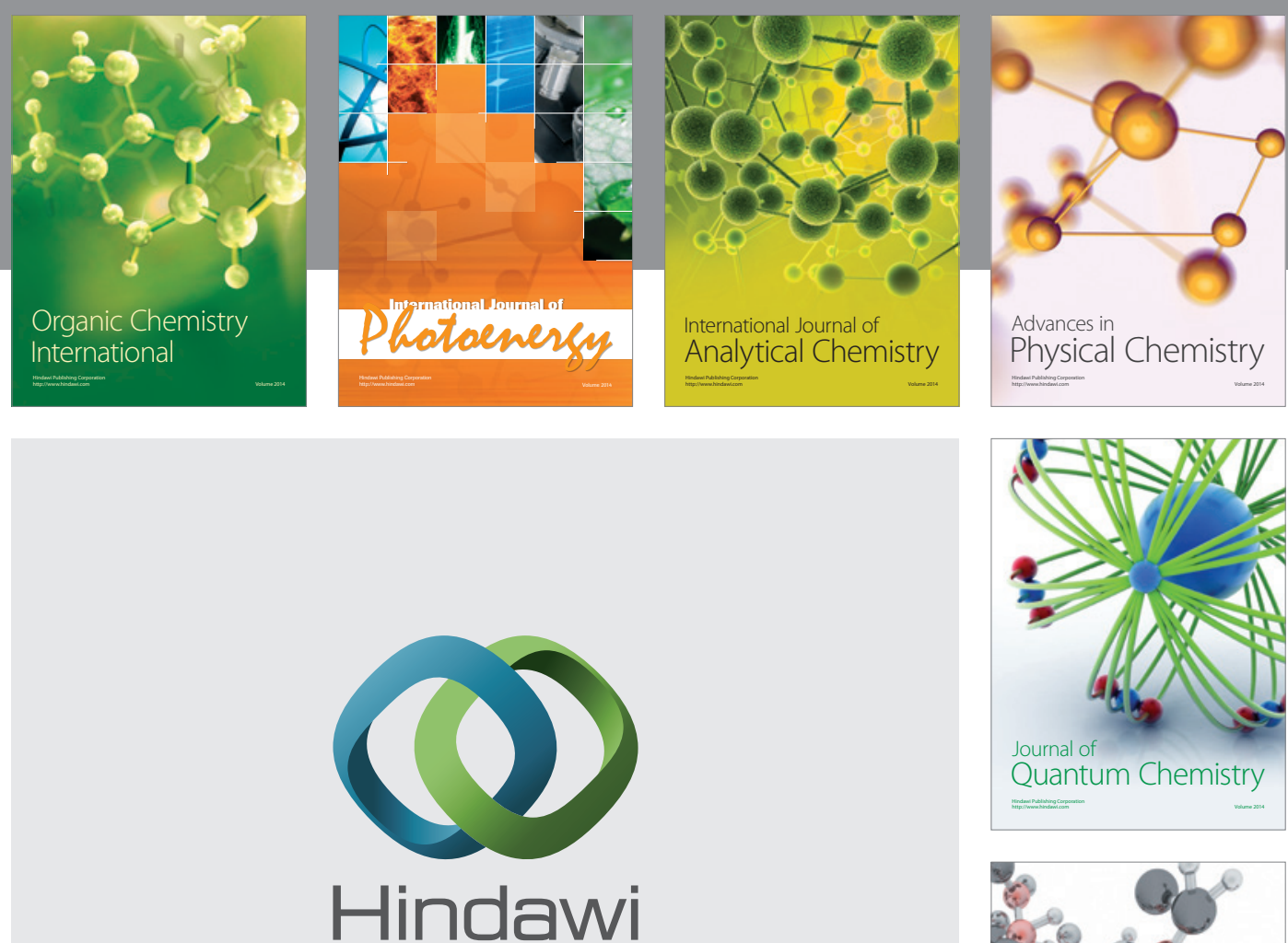

Submit your manuscripts at

http://www.hindawi.com

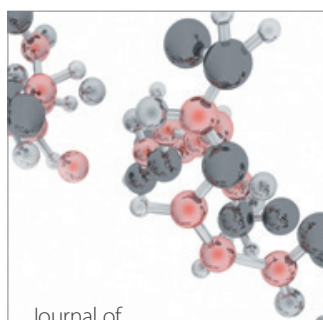

Analytical Methods

in Chemistry

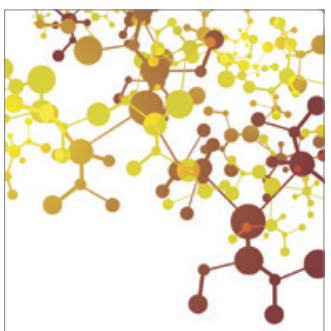

Journal of

Applied Chemistry

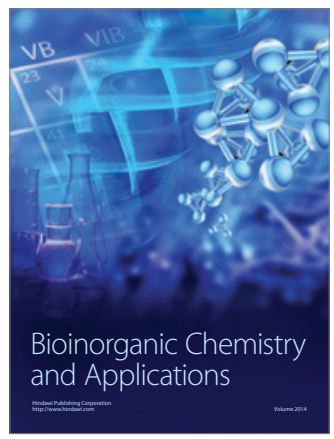

Inorganic Chemistry
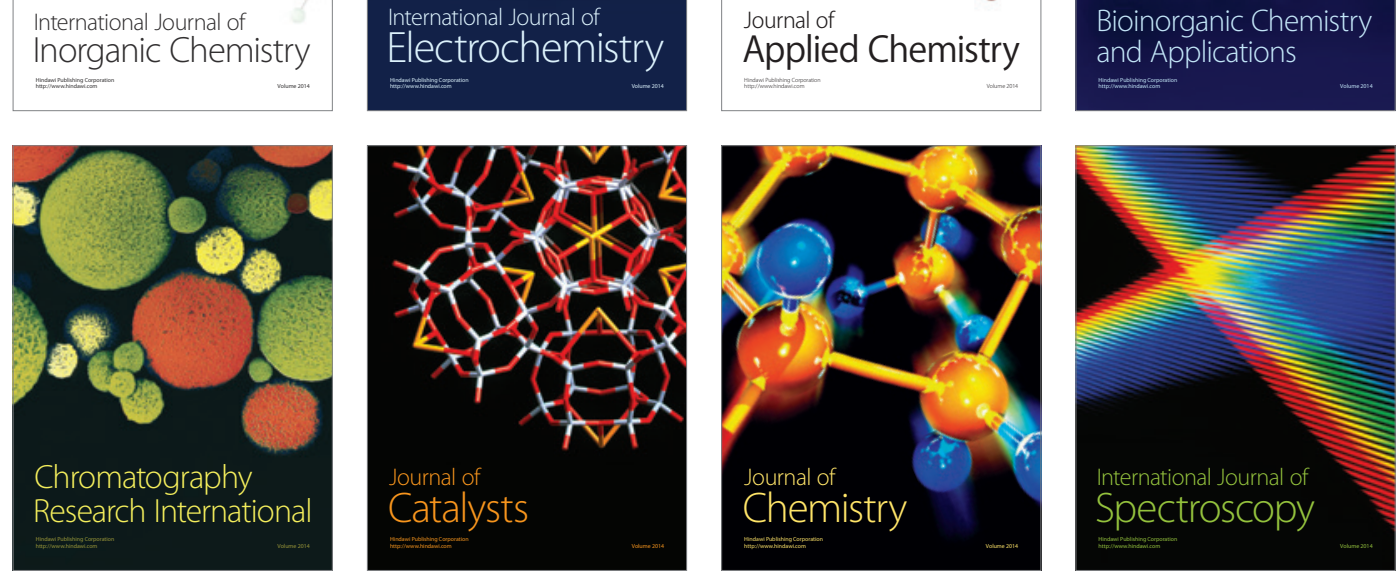